\title{
Function of ssDNA aptamer and aptamer pool against Mycobacterium tuberculosis in a mouse model
}

\author{
FAN CHEN $^{1,2^{*}}$, JING ZHOU ${ }^{3 *}$, YONG-HAO HUANG ${ }^{2}$, FENG-YING HUANG ${ }^{2}$, QIANG LIU $^{2}$, \\ ZHIGANG FANG ${ }^{2}$, SHENG YANG $^{1}$, MIN XIONG $^{1}$, YING-YING LIN $^{2}$ and GUANG-HONG TAN ${ }^{2}$ \\ ${ }^{1}$ Faculty of Life Sciences, Hubei University, Wuchang, Wuhan 430062; \\ ${ }^{2}$ Hainan Provincial Key Laboratory of Tropical Medicine, Pharmacy School, Hainan Medical College, \\ Haikou 571199; ${ }^{3}$ Wuhan Tuberculosis Dispensary, Qiaokou, Wuhan 430030, P.R. China
}

Received July 26, 2012; Accepted November 13, 2012

DOI: $10.3892 / \mathrm{mmr} .2012 .1229$

\begin{abstract}
Novel antibacterial agents against Mycobacterium tuberculosis (MTB) are crucial due to the high infection and mortality rates associated with the disease. Our previous study confirmed that aptamers from a whole bacterium obtained by the Systematic Evolution of Ligands by Exponential Enrichment method specifically bound to the MTB virulent strain $(\mathrm{H} 37 \mathrm{Rv})$. In the present study, the function of aptamers against MTB in a mouse model was further determined. It was demonstrated that the NK2 aptamer has marked inhibitory effects on the adhesion/invasion of H37Rv to macrophages in vitro and stimulates intracellular IFN- $\gamma$ production in $\mathrm{CD}^{+}$ T-cells. The aptamer pool exhibited the strongest inhibitory effect on $\mathrm{H} 37 \mathrm{Rv}$ adhesion/invasion to $\mathrm{CD} 8^{+} \mathrm{T}$-cells in vitro compared with all aptamers-treated and control groups. Histopathological examination of lung biopsy specimens revealed a correlation between aptamer presence and lower pulmonary alveoli fusion, swelling and more prominent air spaces. Acid-fast staining of biopsy specimens from the lungs of the mice demonstrated parallel treatment effects. Results of the present study indicate that the 10th pool aptamers and NK2 may play an active role against H37Rv, however, the effect was different in vivo and in vitro. The treatment effect of 10th pool aptamers was found to be better in comparison to NK2 in vivo. Additional target sites involved in pathogenicity of H37Rv were also revealed and the NK2 binding site and aptamers, including the 10th pool aptamers, may antagonize these sites. Further studies are required to screen for other
\end{abstract}

Correspondence to: Professor Guang-Hong Tan, Hainan Provincial Key Laboratory of Tropical Medicine, Hainan Medical College, No. 3 Xueyuan Road, Haikou 571199, P.R. China

E-mail: scihai@126.com

${ }^{*}$ Contributed equally

Key words: Mycobacterium tuberculosis, ssDNA aptamer, mouse model valuable aptamers which may be used as therapeutic drugs in combination with NK2.

\section{Introduction}

Tuberculosis (TB) caused by Mycobacterium tuberculosis (MTB) has the highest mortality rate worldwide of any infectious disease and has been declared a global health emergency by the World Health Organization $(1,2)$. The attenuated M. bovis Bacillus Calmette Guerin (BCG) is the only available vaccine against TB. However, BCG exhibits varying efficacy (0-80\%) against adult pulmonary TB (3). Emergence of drugresistant isolates of MTB highlight the continued necessity for the discovery and development of drugs active against the bacterium (4-8).

Aptamers have high affinity and specificity for their targets and have been developed for use as oligonucleotide analogs of antibodies. These molecules exhibit several advantages over antibodies and antibiotics. Aptamers are smaller than antibodies and therefore exhibit improved cell penetration, blood clearance and chemical modification. They are also nonimmunogenic and readily synthesized and therefore do not induce an immune host response, which may cause harmful side-effects $(9,10)$. Single-stranded DNA (ssDNA) aptamers exhibit more variable structures, longer relative temperature stability and shelf-life than antibodies and therefore demonstrate significant potential for in vivo use as therapeutics $(11,12)$.

In previous years the Systematic Evolution of Ligands by Exponential Enrichment (SELEX) technique has become increasingly important for the study of protein function, as well as in drug discovery and identification of antagonists against a number of functional proteins $(13,14)$. However, little is known regarding the in vitro SELEX selection strategy utilized for the generation of inhibitors against whole bacteria. Our previous study extracted special aptamers (NK2) and aptamer pools (10th pool) from a whole bacterial SELEX strategy (15) and demonstrated that aptamers inhibit MBT H37Rv invasion of macrophages in vitro (16). In the present study, we further evaluated the function of the 10th pool and NK2 against MBT H37Rv in a mouse model. This investiga- 
tion may aid the development of a new antitubercular agent based on an aptamer species.

\section{Materials and methods}

Bacterial strain and animals. MTB H37Rv (strain ATCC 93009) was purchased from the Beijing Biological Product Institute (Beijing, China). Bacteria were maintained on Lowenstein-Jensen (L-J) medium and harvested in log phase growth. Prior to use, bacilli were washed in $0.05 \%$ Tween- 80 saline and triturated to uniformity. C57BL/6 mice (from the Experimental Building of the Animal Laboratory Center, Wuhan University, Wuhan, China) of either sex were used at 5-6 weeks of age. Bacterial cultures and animal tests were performed in the Animal Biosafety Level 3 Laboratory (ABSL-III) of Wuhan University School of Medicine. The research procedures and the animal protocols in this study were approved by the HPKLTM Ethics \& Animal Use Committee (approval ID: HPKLTME201005).

Fluorescence microscopy and phagocytic index. C57BL/6 mouse peritoneal macrophages were collected and cultured in RPMI-1640 medium with 10\% FBS and penicillin and streptomycin. Following $48 \mathrm{~h}$, cell number was estimated using trypan blue staining. To analyze the effect of aptamers on H37Rv invasion of macrophages, $10^{8} \mathrm{cfu} \mathrm{H} 37 \mathrm{Rv}$ bacteria was incubated with aptamers (pretreated at $85^{\circ} \mathrm{C}$ for $15 \mathrm{~min}$ and then incubated on ice for $3 \mathrm{~min}$ ) at $37^{\circ} \mathrm{C}$ for $15 \mathrm{~min}$ (control group was untreated with aptamer). Following this, centrifugation at $12,000 \mathrm{rpm}$ for 5 min was performed and the supernatant was discarded. Mouse peritoneal macrophages $\left(10^{6}\right)$ were mixed with $10^{8} \mathrm{cfu}$ $\mathrm{H} 37 \mathrm{Rv}$ in $2 \mathrm{ml}$ medium. Phagocytosis was allowed to occur for $1 \mathrm{~h}$ during mixing at $37^{\circ} \mathrm{C}$ in 6 -well costar plates. A pretreated (polylysine, $0.1 \mathrm{mg} / \mathrm{ml}$ ) coverslip was applied to each analytic well. The coverslip was dislodged and fixed by frigorific acetone and then stained with auramine $O$. The coverslip was then observed under a fluorescence microscope (CW4000, Leica, Wetzlar, Germany). Counts of 200 cells from each coverslip were performed and the phagocytic index was calculated using the formula: phagocytic index = (total MTB phagocytosed by macrophage)/(macrophage number of phagocytosed MTB).

Flow cytometry analysis of the effect of aptamers on the invasion of H37Rv to CD4 $4^{+}$and $C D 8^{+} T$ cells. H37Rv $\left(10^{8} \mathrm{cfu}\right.$; prestained with Rhodamine B) was incubated for $1 \mathrm{~h}$ with $10^{6}$ peripheral blood mononuclear cells (PBMCs) collected from the tail vein of C57BL/6 mice. Experimental groups were pretreated with the 10th pool or NK2 as previously described (15). Following this, the cells were washed twice with PBS and incubated with $5 \mu 1$ mouse anti-CD3-PE-Cy5, anti-CD4-FITC (Caltag Laboratories, Buckingham, UK) or isotype control antibodies, at the concentration recommended by the manufacturer's instructions, for $30 \mathrm{~min}$ at $4^{\circ} \mathrm{C}$. The cells were then fixed with $70 \%$ ethanol and analyzed using a fluorescence-activated cell sorter (FACS; Epics Altra II, Beckman Coulter, Miami, FL, USA).

Effect of aptamers on the intracellular expression of IFN- $\gamma$ of $\mathrm{CD}^{+}$and $C D 8^{+} \mathrm{T}$ cells in H37Rv-infected splenocytes. Murine splenocytes $\left(2 \times 10^{6}\right)$ were incubated with $10^{8} \mathrm{cfu} \mathrm{H} 37 \mathrm{Rv}$ or pretreated with the 10th pool and NK2 for $1 \mathrm{~h}$. Following this, $1 \mu \mathrm{g} / \mathrm{ml}$ monensin (eBioscience, San Diego, CA, USA) was added and incubated at $37^{\circ} \mathrm{C}$ for $2 \mathrm{~h}$ in a $5 \%(\mathrm{v} / \mathrm{v}) \mathrm{CO}_{2}$ atmosphere. Cells were washed twice with PBS, resuspended in $100 \mu \mathrm{l}$ cold PBS and incubated with $5 \mu 1$ mouse anti-CD3-PE-Cy5 and antiCD4-FITC antibodies (Caltag Laboratories) at $4^{\circ} \mathrm{C}$ for $30 \mathrm{~min}$. The cells were fixed using $70 \%$ ethanol and incubated with $5 \mu \mathrm{l}$ PE-conjugated mouse anti-IFN- $\gamma$ (Caltag Laboratories) at $37^{\circ} \mathrm{C}$ for $30 \mathrm{~min}$ in the dark. Stained cells were washed with PBS and intracellular cytokine expression of IFN $-\gamma$ in $\mathrm{CD}^{+} \mathrm{CD}^{+}$and $\mathrm{CD}^{+} \mathrm{CD}^{+} \mathrm{T}$ cells was analyzed by FACS.

Challenge infection and analysis of survival rate. C57BL/6 female mice (18-22 g) were used in the study. H37Rv bacteria used for in vivo experiments underwent several passages in C57BL/6 mice to enhance virulence (17). Mice were injected intravenously with $10^{7} \mathrm{cfu} \mathrm{H} 37 \mathrm{Rv} /$ mouse in $0.4 \mathrm{ml}$ saline. Equal MTB was pretreated with $8 \mu \mathrm{g}$ of the 10 th pool or NK2 aptamers, the supernatant was then discarded, and pellets were centrifuged prior to injection with $0.4 \mathrm{ml}$ saline. Mice were fed with standard pelleted food and water for 20 days and each group was composed of 8 mice. Mortality was monitored daily. Survival analysis was analyzed by Kaplan-Meier (SPSS 13.0, Chicago, IL, USA).

Histopathology and acid-fast stain. Lungs or spleens from mice sacrificed 20 days post-infection were homogenized in saline and plated on L-J medium. Following 4-6 weeks incubation at $37^{\circ} \mathrm{C}$, the number of viable organisms in the lungs or spleens was determined. Sections of the lungs and spleens were soaked in $10 \%$ paraformaldehyde for a minimum of $12 \mathrm{~h}$ and examined with hematoxylin and eosin stain and acid-fast stain, respectively.

Statistical analysis. Data were presented as the mean \pm SEM and were analyzed using one-way analysis of variance followed by the Student-Newman-Keuls post-hoc test. $\mathrm{P}<0.05$ was considered to indicate a statistically significant difference.

\section{Results}

Inhibition of MTB invasion of mouse peritoneal macrophages. Compared with untreated, groups pretreated with aptamers (NK2 or 10th pool) inhibited MTB invasion of mouse peritoneal macrophages (Fig. 1A). Similar results were obtained by comparing the phagocytic index with fluorescence microscope observations (Fig. 1B). MTB invasion of mouse peritoneal macrophages was higher in NK2 than the 10th pool.

Inhibition of H37Rv invasion to $C D 3^{+} C D 8^{+} T$ cells. $\mathrm{H} 37 \mathrm{Rv}$ invasion to $\mathrm{CD} 3^{+} \mathrm{CD} 8^{+} \mathrm{T}$ cells was inhibited by the aptamers. Invasion to $\mathrm{CD}^{+} \mathrm{CD} 4^{+} \mathrm{T}$ cells was not inhibited. H37Rv invasion to $\mathrm{CD}^{+} \mathrm{CD}^{+} \mathrm{T}$ cells was found to be significantly decreased from $50.4 \%$ (without aptamers) to 33.9, 28, 21 and $20 \%$ with NK2 aptamer, 10th, 7th and 3rd aptamer pool, respectively (Fig. 2A). These data indicate that aptamer pools protect $\mathrm{CD}^{+} \mathrm{T}$ cells against $\mathrm{H} 37 \mathrm{Rv}$ infection.

Aptamers increase intracellular IFN- $\gamma$ secretion of $\mathrm{CD}^{+}$ $C D 4^{+} T$ cells. Intracellular IFN- $\gamma$ levels in $\mathrm{CD}^{+} \mathrm{CD}^{+}$and 
A
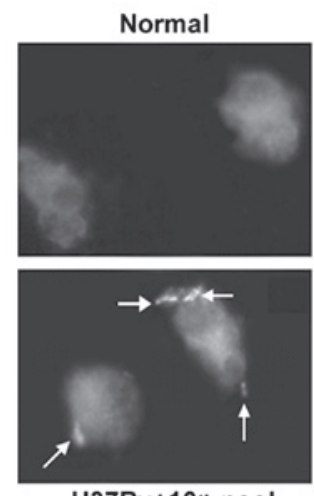

H37Rv+10th pool
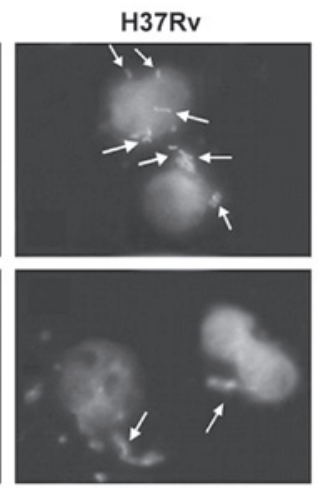

$\mathrm{H} 37 \mathrm{Rv}+\mathrm{NK} 2$
B

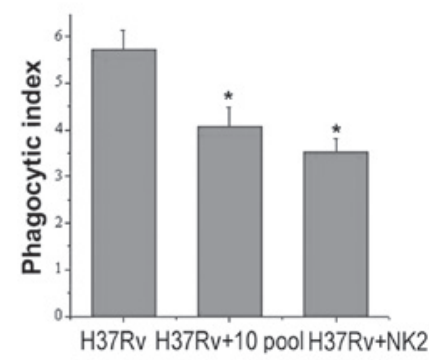

Figure 1. Inhibitory effects of aptamers on H37Rv invasion of murine peritoneal macrophages. (A) Fluorescence microscope images (x1,000), MTB bacteria are labeled with arrows. (B) Quantity analysis of phagocytic index. Data are presented as the mean \pm standard error of the mean (SEM) and were analyzed using one-way analysis of variance followed by the Student-Newman-Keuls post hoc test. "P<0.05, vs. H37Rv. MTB, Mycobacterium tuberculosis.

A
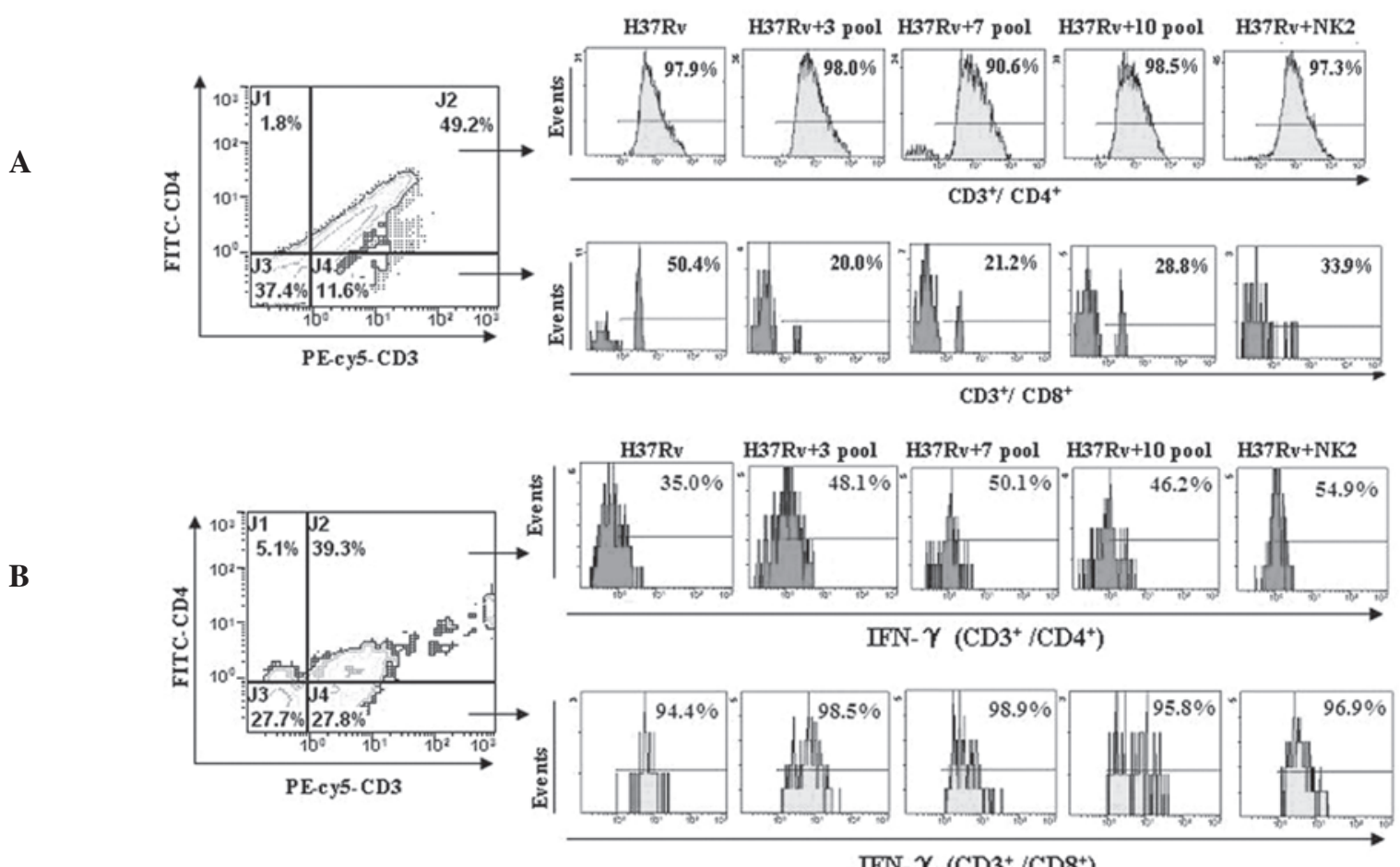

IFN- $\gamma\left(\mathrm{CD}^{+} / \mathrm{CD}^{+}\right)$

Figure 2. Flow cytometry analysis of (A) inhibitory effects of aptamers on H37Rv invasion to $\mathrm{CD}^{+} \mathrm{T}$ cells and (B) intracellular IFN- $\gamma$ levels in CD4 ${ }^{+} \mathrm{T}$ cells

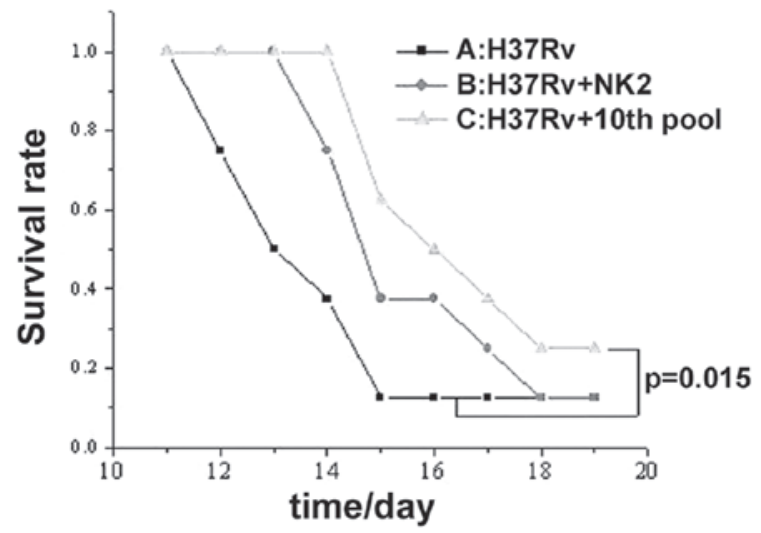

Figure 3. Survival rate following 20-day infection. Kaplan-Meier methods were used to calculate the survival analyses.
$\mathrm{CD}^{+} \mathrm{CD} 8^{+} \mathrm{T}$ cells were detected by flow cytometry. When PBMCs from mice were infected by H37Rv in vitro, increased levels of intracellular IFN- $\gamma$ were observed in the presence of aptamers in $\mathrm{CD}^{+} \mathrm{CD}^{+} \mathrm{T}$ cells, but not in $\mathrm{CD}^{+} / \mathrm{CD}^{+}$ $\mathrm{T}$ cells. Intracellular IFN- $\gamma$ levels in $\mathrm{CD} 3^{+} \mathrm{CD} 4^{+} \mathrm{T}$ cells were increased from $35 \%$ (without aptamers) to $48.1,50.1,46.2$, 53.6 and $54.9 \%$ with $3 \mathrm{rd}, 7$ th, 10th and 12th aptamer pools and NK2 aptamer, respectivly. The NK2 aptamer had the strongest stimulatory effect on intracellular IFN- $\gamma$ levels in $\mathrm{CD}^{+}$ $\mathrm{CD}^{+} \mathrm{T}$ cells (Fig. 2B). These data indicate that aptamers, particularly the NK2 aptamer, stimulate IFN $-\gamma$ production and decrease the infection efficiency of MTB.

Treatment of H37Rv in vivo. Effect of 10th round aptamer pool or NK2 aptamer on acute tuberculosis in mice was 


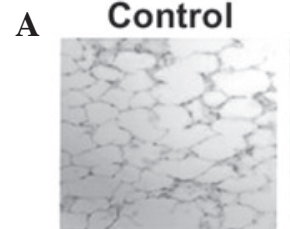

$\mathbf{B}$

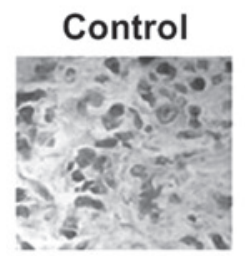

H37Rv

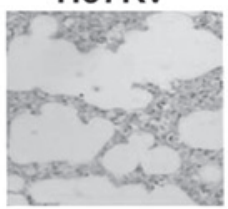

H37Rv

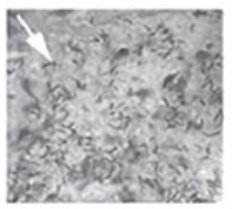

$\mathrm{H} 37 \mathrm{Rv}+\mathrm{NK} 2 \mathrm{H} 37 \mathrm{Rv}+10$ pool
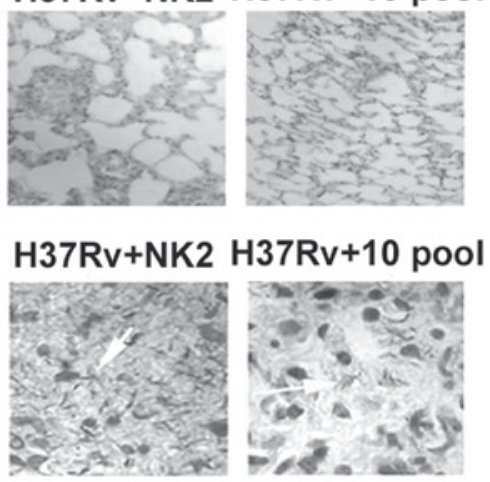

C

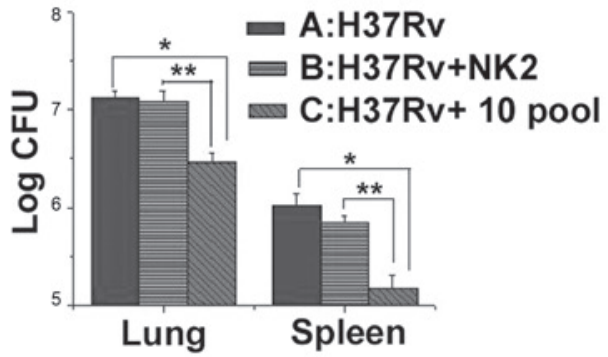

Figure 4. (A) Histopathological examination of biopsy specimens from lungs of mice (HE, x40) and (B) acid fast stain of biopsy specimens from lungs of mice (x1,000), MTB bacteria are labeled with arrows. (C) Determination of CFU from lung and spleen of infected mice with or without aptamers treatment. Colony counts were determined after 4-weekly incubation at $37^{\circ} \mathrm{C}$ and presented in $\log$ cfu. Data are presented as the mean \pm SEM and were analyzed using one-way analysis of variance (ANOVA) followed by the Student-Newman-Keuls post hoc test. " P<0.05 vs. H37Rv; ${ }^{* *} \mathrm{P}<0.01$, vs. H37Rv + NK2. MTB, Mycobacterium tuberculosis.

examined. In the control group, $\mathrm{H} 37 \mathrm{Rv}$ injection for 13 days, resulted in $50 \%$ mortality. Survival rate of the mice was prolonged for 2 days with a single injection of aptamer NK2 and 3 days with a single injection of aptamer pool (10th round selection; $\mathrm{P}<0.05$; Fig. 3). Histopathological examination of lungs from mice injected with $\mathrm{H} 37 \mathrm{Rv}$ revealed a marked acute inflammatory reaction compared with normal mice. NK2- or aptamer pool treated-mice demonstrated decreased pulmonary alveoli fusion and swelling and more prominent air spaces, similar to lungs of normal mice (Fig. 4A). Furthermore, compared with normal mice the density of acid fast bacillus in the lung was higher in control mice than mice that had received a single injection of aptamer NK2 or 10th pool aptamers (Fig. 4B). Number of H37Rv colonies was higher in control mice than in the 10th aptamer pool or NK2 groups (Fig. 4C). The present study revealed that, in vivo, the 10th pool of aptamers and NK2 had an active therapeutic effect on H37Rv infection and the 10th pool of aptamers was more effective than NK2.

\section{Discussion}

The use of aptamers as therapeutic drugs has been reported in numerous research fields, including HIV (18) and cancer (19). Aptamers may inhibit MTB infection through blockage of virulence components or epitopes of $\mathrm{H} 37 \mathrm{Rv}$. In vitro, inhibition of H37Rv macrophage infection by NK2 aptamers was higher when compared with the 10th aptamer pool (Fig. 1), however, the 10th aptamer pool prolonged the survival rate of mice, enhancing clearance of the bacterium in vivo to a more significant degree (Figs. 3 and 4). This may be due to the complex surface of MTB and the degradation of DNA aptamers by nuclease enzymes (20). The 10th aptamer pool contained various non-special aptamers that may aid resistance to nucleases and function in synergy with the NK2 aptamer. Thus, the 10th aptamer pool was more effective than the NK2 aptamer in vivo. Previously, modified aptamers with longer half-lives were developed to resist nuclease digestion. However, disadvantages have been identified, including an increased rate of integration into the chromosomal DNA of host $\mathrm{T}$ cells. There are multiple binding sites and antigen epitopes on the surface of H37Rv and synergism of several aptamers is required to inhibit $\mathrm{H} 37 \mathrm{Rv}$ infection. In the present study, we noted that inhibition of $\mathrm{H} 37 \mathrm{Rv}$ invasion to $\mathrm{CD} 8^{+} \mathrm{T}$ cells decreased as the screening process progressed (Fig. 2A). This observation may be due to inhibitory aptamers being missed through SELEX. This may be explained by important components in the reverse target (BCG) which stimulate the cytotoxic T-cell effect. Currently, BCG is the only vaccine against tuberculosis, however, its immune protective effects are not always effective. A possible explanation for reduced efficacy may be due to the effect of aptamers on $\mathrm{CD}^{+}$and $\mathrm{CD}^{+} \mathrm{T}$ cells (Fig. 2).

The present study demonstrates that the aptamer pool and NK2 aptamer exhibit protection against tuberculosis, however, their mechanisms of action are different. NK2 revealed improved inhibition of H37Rv invasion to mice peritoneal macrophages and stimulation of $\mathrm{CD}^{+}{ }^{+} \mathrm{T}$-cell INF- $\gamma$ secretion compared with the 10th pool. However, survival rate and histological analysis revealed that the 10th pool has a better therapeutic effect compared with NK2. The results demonstrated we should not only pay attention to those aptamers in the majority (including NK2), but also investigate the role of early deserted aptamers. The present study demonstrates the limitations of ssDNA aptamers, highlighting a number of factors which must be considered to avoid available aptamer loss.

\section{Acknowledgements}

This study was supported by Wuhan Health Bureau Funded Projects (WH12A03) and the Hainan Natural Science Fund (nos. 808162 and 812199).

\section{References}

1. Raviglione MC, Dye C, Schmidt S and Kochi A: Assessment of worldwide tuberculosis control. WHO global surveillance and monitoring project. Lancet 350: 624-649, 1997. 
2. Dye C, Scheele S, Dolin P, Pathania V and Raviglione MC: Consensus statement. Global burden of tuberculosis: estimated incidence, prevalence and mortality by country. WHO Global Surveillance and Monitoring Project. JAMA 282: 677-686, 1999.

3. Fine PE: Variation in protection by BCG: implications of and for heterologous immunity. Lancet 346: 1339-1345, 1995.

4. Pablos-Mendez A, Raviglione MC, Laszlo A, Binkin N, Rieder HL,BustreoF, Cohn DL,Lambregts-van Weezenbeek CS, Kim SJ, Chaulet P and Nunn P: Global surveillance for antituberculosis-drug resistance 1994-1997. World Health Organization-International Union against tuberculosis and lung disease working group on anti-tuberculosis drug resistance surveillance. N Engl J Med 338: 1641-1649, 1998.

5. Ahmed N and Hasnain SE: Genomics of Mycobacterium tuberculosis: old threats and new trends. Indian J Med Res 120: 207-212, 2004.

6. Baptista IMFD, Oelemann MC, Opromolla DVA and Suffys PA: Drug resistance and genotypes of strains of Mycobacterium tuberculosis isolated from human immunodeficiency virusinfected and non-infected tuberculosis patients in Bauru, São Paulo, Brazil. Mem Inst Oswaldo Cruz: 97: 1147-1152, 2002.

7. Pereira M, Tripathy S, Inamdar V, Ramesh K, Bhavsar M, Date A, Iyyer R, Acchammachary A, Mehendale S and Risbud A: Drug resistance pattern of Mycobacterium tuberculosis in seropositive and seronegative HIV-TB patients in Pune, India. Indian J Med Res 121: 235-239, 2005.

8. Yew WW and Leung CC: Update in tuberculosis 2007. Am J Respir Crit Care Med 177: 479-485, 2008.

9. Jayasena SD: Aptamers: An emerging class of molecules that rival antibodies in diagnostics. Clin Chem 45: 1628-1650, 1999.

10. Guthrie JW, Hamula CLA, Zhang H and Le XC: Assays for cytokines using aptamers. Methods 38: 324-330, 2006.

11. Ulrich H, Alves MJM and Colli W: RNA and DNA aptamers as potential tools to prevent cell adhesion in disease. Braz J Med Bio 34: 295-300, 2001.
12. Marimuthu C, Tang TH, Tominaga J, Tan SC and Gopinath SC: Single-stranded DNA (ssDNA) production in DNA aptamer generation. Analyst 137: 1307-1315, 2012

13. Shimada T, Fujita N, Maeda M and Ishihama A: Systematic search for the Cra-binding promoters using genomic SELEX system. Genes Cells 10: 907-918, 2005.

14. Jensen KB, Atkinson BL, Willis MC, Koch TD and Gold L: Using in vitro selection to direct the covalent attachment of human immunodeficiency virus type $1 \mathrm{Rev}$ protein to high-affinity RNA ligands. Proc Natl Acad Sci USA 92: 12220-12224, 1995.

15. Chen F, Zhou J, Luo F, Mohammed AB and Zhang ZL: Aptamer from whole-bacterium SELEX as new therapeutic reagent against virulent Mycobacterium tuberculosis. Biochem Biophys Res Commun 357: 743-748, 2007.

16. Chen F, Zhang XL, Zhou J, Liu S and Liu J: Aptamer inhibits Mycobacterium tuberculosis (H37Rv) invasion of macrophage. Mol Biol Rep 39: 2157-2162, 2012.

17. Jagannath C, Emanuele MH and Hunter RL: Activity of poloxamer CRL-1072 against drug-sensitive and resistant strains of Mycobacterium tuberculosis in macrophages and in mice. Intern J Anti Agent 15: 55-63, 2000.

18. Jing $\mathrm{N}$ and Hogan ME: Structure-activity of tetrad-forming oligonucleotides as a potent anti-HIV therapeutic drug. J Biol Chem 273: 34992-34999, 1998.

19. Blank M, Weinschenk T, Priemer M and Schluesener $H$ Systematic evolution of a DNA aptamer binding to the rat brain tumor microvessels: selective targeting of endothelial regulatory protein pigpen. J Biol Chem 276: 16464-16468, 2001

20. Cerchia L, Ducongé F, Pestourie C, Boulay J, Aissouni Y, Gombert K, Tavitian B, de Franciscis V and Libri D: Neutralizing aptamers from whole-cell SELEX inhibit the RET receptor tyrosine kinase. PLoS Biol 3: e123, 2005. 